\title{
Vascular Thoracic Outlet Syndrome: Experiences at the National Institute of Cardiovascular Diseases, Dhaka
}

MF Hossain, SR Sarker, MN Sabah, AHM Bashar, NK Dey, E Hakim, GMM Hossain, NC Mandal

Department of Vascular Surgery, NICVD, Dhaka

\begin{abstract}
Keywords:

Thoracic outlet

syndrome, Aneurysm, Thromboembolism.

Background: To analyze our recent experiences at the National Institute of Cardiovascular Diseases (NICVD) with the management of vascular Thoracic Outlet Syndrome (TOS).

Methods: Retrospective review identified 51 patients (age range 23 to 67 years) with vascular TOS, among them 47 patients with arterial type TOS underwent surgical treatment from January 2012 to December 2015. Evaluation included clinical assessment plus duplex ultrasonography and contrast arteriography and outcome of treatment.

Results: In 44 (94\%) patients, surgery was successful in relieving the ischemic symptoms. Major amputations could be avoided in 21 of the 28 patients presenting with gangrene. Recurrent thrombo-embolism of the distal arterial tree was found in 5 patients requiring repeat embolectomy. At one year follow-up, $73 \%$ of the patients remained free from ischemic upper limb symptoms.

Conclusions: Though relatively uncommon, arterial TOS is a potentially limb threatening condition. Early detection of the anatomical abnormality with its surgical correction is required to avoid major complications.
\end{abstract}

(Cardiovasc. j. 2018; 11(1): 50-52)

\section{Introduction:}

Thoracic outlet is the gateway for the neurovascular structures from the thorax and neck to exit into the upper extremity. The main structures that pass through this space are the Brachial nerve plexus, the Subclavian artery (SCA) and the Subclavian vein. ${ }^{1}$ Thoracic Outlet Syndrome (TOS) refers to the pathologic conditions caused by compression of these neurovascular structures at the thoracic outlet area. It has been reported that more than $95 \%$ of the TOS patients present with neurological symptoms due to compression of the nerves of the brachial plexus. ${ }^{2}$ Most of the patients with arterial TOS are referred to vascular centers because of symptoms of upper limb ischemia resulting from thromboembolism secondary to compression injury to the SCA. Such compression of the SCA may be due to the presence of a cervical rib, anomalous first rib, fibrous band attached to the tip of the rudimentary cervical rib and sometimes an unusually tight anterior scalene muscle. ${ }^{3}$
Unilateral ischemia of fingers or handespecially in young patients-should be considered first of all as a peripheral manifestation of a cervical rib-syndrome. ${ }^{4}$ These patients usually require surgical intervention at the thoracic outlet area to relieve the compression on the artery and repair the damaged SCA, when required. ${ }^{5}$

Venous TOS, on the other hand, present with pain and swelling of the affected upper limb resulting from Axillary-Subclavian vein thrombosis leading to venous hypertension in the upper limb. The well-described Paget Schroetter Syndrome refers to the effort thrombosis of the Axillary-Subclavian vein usually secondary to repetitive unaccustomed use of the arm in addition to the presence of the compressive structures at the thoracic outlet. ${ }^{6}$ The National Institute of Cardiovascular Diseases and Hospital (NICVD) is one of the two tertiary referral centers for vascular diseases that cater to a population of about 160 millions. We undertook the present study to analyze our

Address of Correspondence: Dr. Md. Fidah Hossain, Department of Vascular Surgery, National Institute of Cardiovascular Diseases, Dhaka, Bangladesh. E-mail: fidah.hossain@gmail.com 
experiences with vascular TOS over the last 4 years at the NICVD.

\section{Methods:}

Between January 2012 and December 2015, 51 patients with vascular manifestations of TOS were referred to the vascular outpatient department or vascular emergency department of the NICVD. History, clinical presentation and imaging studies led to a diagnosis of arterial type TOS in 47 and venous type TOS in 4 patients.

Diagnostic Work-up:

Diagnostic work-up included plain digital radiogram of the cervical spine, Duplex ultrasound examination of the upper limb vasculature and CT angiogram of the upper limb vessels. Cervical rib which was clearly seen on radiogram was identified as the causative anatomical abnormality in 36 patients which included one patient having regeneration of the cervical rib 11 years after an incomplete excision. Generally, radiography was adequate in identifying cervical and anomalous first ribs. In 3 patients Magnetic Resonance Imaging (MRI) was used to identify fibrous band at the tip of the rudimentary cervical rib. Duplex ultrasound scan revealed an aneurysmal Subclavian artery (SCA) in 29 patients and thrombo-embolic occlusion of the distal arterial tree in 31 patients. $\mathrm{CT}$ angiogram was done in 11 patients to better delineate the vascular anatomy of the upper limb.

Operative Strategy and Surgical Technique: Operative treatment was done in all 47 patients of arterial TOS. The strategy was to remove the causative anatomical defect which was done by excision of cervical rib, anomalous $1^{\text {st }}$ rib or fibrous band. Repair of the SCA aneurysm was done when present. Fogarty embolectomy was also done whenever there was evidence of distal thrombo-embolism.

Follow-Up:

All patients were followed up at the vascular outpatient department of NICVD at an interval of 1 month, 3 months, 6 months and then annually. In each visit, patients were subjected to physical examination with particular attention to vascular and nervous system, radiological examination and Duplex vascular studies.

\section{Results:}

Between January 2012 and December 2015, 47 patients with arterial type TOS underwent surgical treatment. Twenty eight patients presented with acute or subacute critical upper limb ischemia with gangrene. The remaining 19 patients had ischemic symptoms without tissue loss. In 36 patients, cervical rib was identified as the causative anatomical abnormality. Abnormal $1^{\text {st }}$ rib was found in 3 patients. In 29 patients, the Subclavian artery (SA) was aneurysmal. Thrombo-embolic occlusion of the distal arterial tree was noted in 31 patients. Surgical strategy was aimed at decompression of the thoracic outlet and included excision of the cervical rib $(n=36)$, anomalous first rib $(n=3)$, fibrous band $(n=8)$ and scalenectomy $(n=47)$. Repair of the aneurymal SA was done in 29 cases. Fogarty embolectomy was performed in 23. All operations were done through a supraclavicular approach.

In $44(94 \%)$ patients, surgery was successful in relieving the ischemic symptoms. Major amputations could be avoided in 21 of the 28 patients presenting with gangrene. Recurrent thrombo-embolism of the distal arterial tree was found in 5 patients requiring repeat embolectomy. At one year follow-up, $73 \%$ of the patients remained free from ischemic upper limb symptoms.

\section{Discussion:}

As the overwhelming majority of patients of TOS belong to the neurogenic type, vascular surgical expertise is sought only when there is involvement of vascular structures at the thoracic outlet area. Patients with SCA compression present with acute or subacute upper extremity ischemic symptoms of varying severity. In the present study, more than 50\% of the patients presented with advanced ischemia with tissue loss. Generally, this was the consequence of delay in diagnosis and referral. The average time between the onset of symptoms and referral in the present series was 8.4 days. Cervical rib was the main pathological factor in this series found in 36 patients. Many previous reports also identify cervical rib and or anomalous first rib as the causative factor for TOS. ${ }^{7}$ Likewise, resection 
of the cervical or anomalous first rib is an important step of surgery in these patients. Generally, this can be adequately done through a transaxillary approach. ${ }^{8}$ However, we preferred a supraclavicular approach since most of our patients had SCA aneurysm that needed to be repaired. Indeed, supraclavicular approach has been found to be more convenient when SCA aneurysm repair is needed. 9 Whether or not aneurysmal SCA should be routinely repaired is a contentious issue. It is thought that smaller aneurysms resolve spontaneously once the causative anatomical defect has been corrected and normal hemodynamics has been restored. ${ }^{10}$ Need to be repaired a surgical strategy for patients with arterial TOS generally conforms to the reported standards for this disease entity. ${ }^{11}$ We prefer supraclavicular approach because it offers complete exposure of the subclavian artery, cervical and first ribs.

\section{Conclusions:}

Vascular TOS is seen less frequently than the neurogenic form; however, in most cases it requires surgical treatment. Arterial TOS is a potentially limb threatening condition which can best be avoided by early recognition, diagnosis and treatment. Early detection of the anatomical abnormality with its surgical correction is required to avoid major complications.

Conflict of Interest - None.

\section{References:}

1. Chang KZ, Likes K, Davis K, Demos J, Freischlag JA. The significance of cervical ribs in thoracic outlet syndrome. $J$ Vasc Surg 2013; 57(3):771-775.

2. Davidovic LB, Kostic DM, Jakovljevic NS, Kuzmanovic IL, Simic TM. Vascular thoracic outlet syndrome. World J Surg 2003; 27(5):545-550.

3. Sanders RJ. Thoracic Outlet Syndrome: General Considerations. In: Cronewell JL, Johnston KW. Eds. Rutherford's Vascular Surgery. Philadelphia: Saunders Elsvier; 2010. 1865-1917.

4. Heyden B, Vollmar J. Thoracic outlet-syndrome with vascular complications. J Cardiovasc Surg (Torino). 1999; 20(6):531-536.

5. Criado E, Berguer R, Greenfield L. The spectrum of arterial compression at the thoracic outlet. J Vasc Surg 2010; 52(2):406-411.

6. Durham JR, Yao JS, Pearce WH, Nuber GM, McCarthy WJ 3rd. Arterial injuries in the thoracic outlet syndrome. J Vasc Surg 1995; 21(1):57-69.

7. Wise R. Seventh cervical rib associated with subclavian artery occlusion and multiple infarcts: case report. $J$ Neurosci Nurs 2008;40(3):169-172.

8. Engel A, Adler OB, Carmeli R. Subclavian artery aneurysm caused by cervical rib: case report and review. Cardiovasc Intervent Radiol 1989; 12(2):92-94.

9. Sen S, Di ${ }^{\circ}$ çigil B, Boga M, Ozkisacik E, Inci I. Thoracic outlet syndrome with right subclavian artery dilatation in a child - transaxillary resection of the pediatric cervical rib. Thorac Cardiovasc Surg 2007; 55(5):339-341.

10. Urschel HC Jr, Razzuk MA. Neurovascular compression in the thoracic outlet: changing management over 50 years. Ann Surg 1998; 228(4):609-617.

11. Sanders RJ, Haug C. Review of arterial thoracic outlet syndrome with a report of five new instances. Surg Gynecol Obstet 1991; 173(5):415-425. 